\title{
A De Sitter-Hubble Equation, the Force in Special Relativity and Some Concepts on Symmetry
}

\author{
Juan G. Lartigue* \\ Faculty of Chemistry, National University of Mexico, Mexico City, Mexico \\ Email: jmlg5@hotmail.com
}

How to cite this paper: Lartigue, J.G. (2018) A De Sitter-Hubble Equation, the Force in Special Relativity and Some Concepts on Symmetry. Journal of Modern Physics, 9, 1317-1325.

https://doi.org/10.4236/jmp.2018.96080

Received: April 9, 2018

Accepted: May 27, 2018

Published: May 30, 2018

Copyright $\odot 2018$ by author and Scientific Research Publishing Inc. This work is licensed under the Creative Commons Attribution International License (CC BY 4.0).

http://creativecommons.org/licenses/by/4.0/

\begin{abstract}
Previous works [1] [2] proposed several relevant characteristics of the Hubble field such as the intensity $\Gamma_{\mathrm{H}}$, potential $\mathrm{V}_{\mathrm{H}}$, force $\mathrm{F}_{\mathrm{H}}$, Poisson equation, energy $\mathrm{E}_{\mathrm{H}}$ and a tensor $\left(\mathrm{T}_{\mu \nu}\right)_{\mathrm{H}}$ as well as Ricci and Hubble scalars. In this paper, it is made a proposal for two new ones: the kind of the Hubble field as well as a De Sitter-Hubble equation. Moreover, it is proposed a necessary change in the equation of force in Special Relativity. It is included a paragraph on the search for Symmetry in Physics, Chemistry and Time. Conclusions mention an eventual mirror image both the intensity of the Hubble and gravitational fields as well as the relative impossibility for a Big Crunch.
\end{abstract}

\section{Keywords}

Hubble Field, Relativistic Force, De Sitter Equation, Symmetry

\section{Introduction}

Two previously proposed concepts in references [1], [2] were the equations for the constant acceleration in the Universe expansion $\Gamma_{\mathrm{H}}$ and its velocity $\mathbf{v}_{\mathrm{H}}$ (whose numerical values have been corrected in this version). Other concepts will be referred, if necessary in the text, to develop two new topics: a De Sitter-Hubble equation and the time Symmetry.

\subsection{The Kind of the Hubble Field}

Since it is possible to assign a scalar Hubble positive potential $\mathrm{V}_{\mathrm{H}}\left(\mathrm{m}^{2} \cdot \mathrm{s}^{-2}\right)$ to every point in the expanding Universe ([2], Equation (1.1)), it may be assumed that the Hubble field is a positive scalar field, though its spatial derivative renders,

${ }^{\star}$ Professor (retired). 
at every point, the field intensity or the Hubble acceleration vector:

$$
\Gamma_{H}=\nabla V_{H}\left(\mathrm{~m} \cdot \mathrm{s}^{-2}\right)
$$

Besides, this was obtained as a function of the Hubble parameter, as:

$$
\Gamma_{\mathrm{H}}=\mathrm{H}^{2} \cdot \mathbf{r}\left(\mathrm{m} \cdot \mathrm{s}^{-2}\right)=\text { a universal constant }
$$

which represents the constant acceleration of the Universe expansion. Some corrections have been made in the numerical values of the Hubble and the gravitational present intensities; so, it gives $\Gamma_{\mathrm{H}}=2.0 \times 10^{-9}\left(\mathrm{~m} \cdot \mathrm{s}^{-2}\right)$ and $\Gamma_{\mathrm{G}}=2.7 \times 10^{-9}$ $\left(\mathrm{m} \cdot \mathrm{s}^{-2}\right)$; (the last figure was calculated as the intensity of the gravitational field at the radial Universe function). Both fields are, supposedly, present in the total Universe, i.e., in the observable as well as in the external empty space. The source of a gravitational field is the only presence of mass, which is detected by the movements of other masses. The Hubble field has not a known source; its potential $\mathrm{V}_{\mathrm{H}}$ is universal and it is also detected by the movement of masses. The intensity of the Hubble field is an outward oriented vector, which represents the Universe expansion. The net expansion acceleration, $\boldsymbol{\Gamma}_{\mathrm{U}}$ expressed in reference ([1] Equation (3.3)) as the difference between both $\Gamma$ 's, has been discarded since they do not interact in practice, each one acts independently: in fact, $\boldsymbol{\Gamma}_{\mathrm{G}}$ acts exclusively on the Universe's mass attraction and $\boldsymbol{\Gamma}_{\mathrm{H}}$ does on the space expansion. So the critical point, a casual equality of $\boldsymbol{\Gamma}_{\mathrm{H}}$ and $\boldsymbol{\Gamma}_{\mathrm{G}}$, has not any physical meaning (Figure 1).

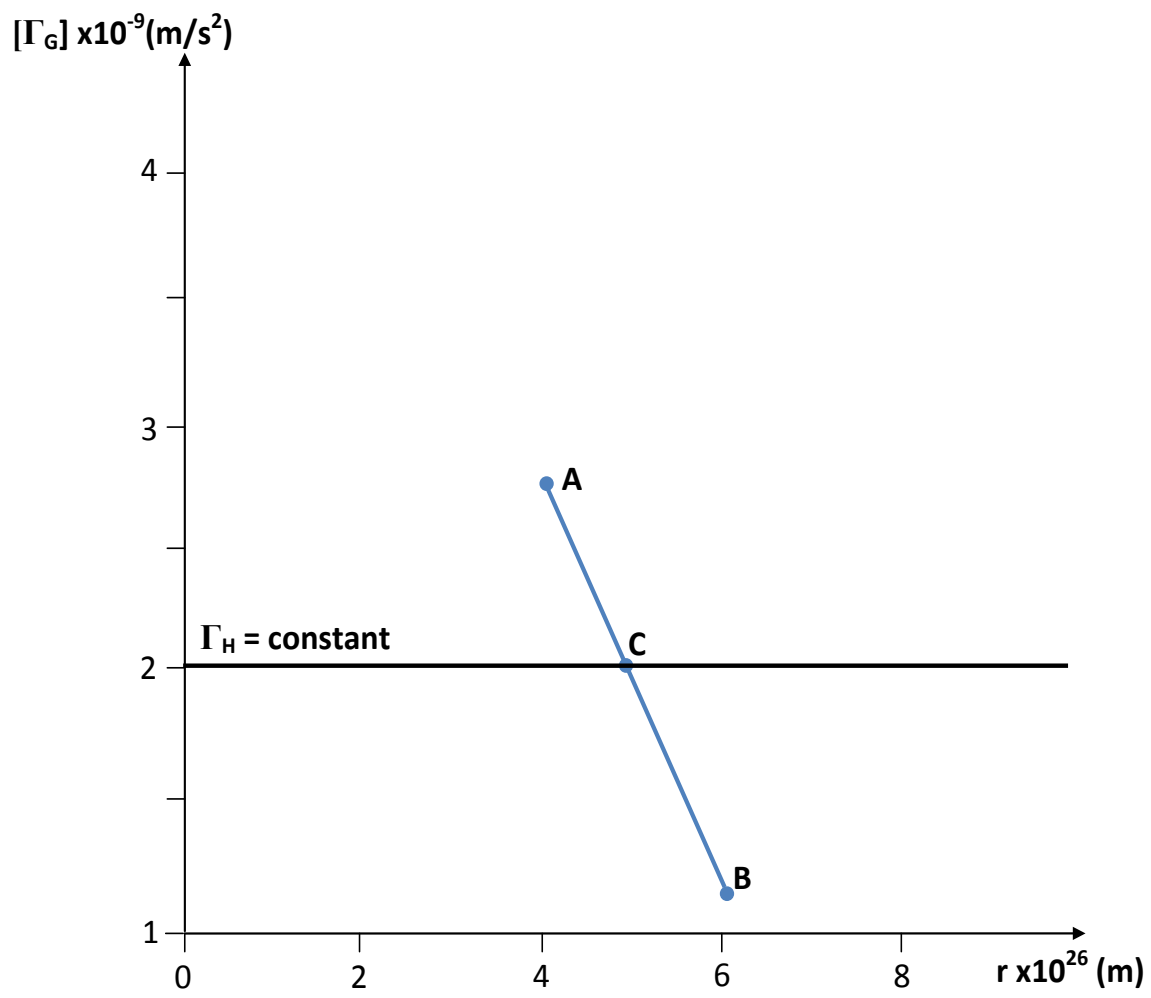

Figure 1. $\Gamma_{\mathrm{H}}$ and $\left[\Gamma_{\mathrm{G}}\right]$ as functions of the coordinate $\mathrm{r}$. A $\left(\mathrm{r}=4 \times 10^{26} \mathrm{~m},\left[\Gamma_{\mathrm{G}}\right]=2.7 \times 10^{-9}\right.$ $\left.\mathrm{m} / \mathrm{s}^{2}\right) ; \mathrm{B}\left(\mathrm{r}=6 \times 10^{26} \mathrm{~m},\left[\Gamma_{\mathrm{G}}\right]=1.2 \times 10^{-9} \mathrm{~m} / \mathrm{s}^{2}\right) ; \mathrm{C}\left(\mathrm{r}=4.4 \times 10^{26} \mathrm{~m},\left[\Gamma_{\mathrm{G}}\right]=2.0 \times 10^{-9} \mathrm{~m} / \mathrm{s}^{2}\right.$ $\left.=\Gamma_{\mathrm{H}}\right)$. 


\subsection{The Linear Velocity in the Hubble Expansion}

Assuming that each portion of the matter in the Universe corresponds to a particular co-moving spherical coordinate $(\mathbf{r}, \theta, \phi)$, all of the masses are being accelerated at a $\Gamma_{\mathrm{H}}$ value in their respective coordinates with a particular velocity that is a function of their life-time, independent of the peculiar velocities involved. So, the expansion velocity of the Universe is:

$$
\mathbf{v}=\Gamma_{\mathrm{H}} \cdot \mathrm{t}_{\mathrm{o}}\left(\mathrm{m} \cdot \mathrm{s}^{-2}\right)
$$

Therefore, in the probable case that the total matter in the Universe would have been formed at the Big Bangtime $(t=0)$, each cluster would be moving, at the present time $\left(\mathrm{t}_{\mathrm{o}}\right)$ in its own co-moving coordinate, at an actual speed $\mathbf{v}$, near c. If the creation continues till a time $t_{i}$, the new matter would be pulled by the acceleration $\Gamma_{\mathrm{H}}$, though its velocity $\mathbf{v}_{\mathrm{i}}$ must correspond to its life time:

$$
\mathbf{v}_{\mathrm{i}}=\Gamma_{\mathrm{H}}\left(\mathrm{t}_{\mathrm{o}}-\mathrm{t}_{\mathrm{i}}\right)\left(\mathrm{m} \cdot \mathrm{s}^{-1}\right)
$$

In the case that the total creation was at $\mathrm{t}=0$, the present expansion velocity would be $\mathbf{v}_{\mathrm{o}} \boldsymbol{c} 1.3 \mathrm{c}$. Since in co-moving coordinates it is not applied the concept of a reference frame, the above mentioned velocities could be assumed as relative, only, to the Big Bang position in the four dimensional space $(\mathrm{r}=\mathrm{\theta}=\phi=\mathrm{t}=$ $0)$.

\section{A Proposed Correction for the Relativistic Equation of Force}

In reference ([2], Equation (2.5)), it was tried to show that, by means of its generalized coordinates, the Hubble force would be a constant of movement. Given that the Hubble force is not originated by a matter presence, it would be better expressed by means of the intensity of the Hubble field $\Gamma_{\mathrm{H}}$ defined as the gradient of the Hubble potential in Equation (1) or, experimentally, by an acceleration that could be evaluated by spectroscopic or CMB methods. In the classical case the first two Newton laws are:

$$
\begin{aligned}
\mathbf{p} & =\mathrm{M} \mathbf{v} \\
\mathbf{F} & =\mathrm{M} \cdot \mathbf{a}
\end{aligned}
$$

The acceleration is defined as:

$$
\mathbf{a}=\frac{\mathrm{d} \mathbf{v}}{\mathrm{dt}}
$$

In the Special Theory of Relativity, the momentum is:

$$
\mathbf{p}^{*}=\mathrm{Mv} \gamma
$$

where

$$
\gamma=\left(1-\beta^{2}\right)^{-1 / 2}
$$

and 


$$
\beta=\frac{\mathrm{v}}{\mathrm{c}}
$$

Therefore, the force would be:

$$
\mathbf{F}^{*}=\frac{\mathrm{Mvd} \gamma}{\mathrm{dt}}+\frac{\mathrm{M} \gamma \mathrm{d} \mathbf{v}}{\mathrm{dt}}
$$

which has been assumed as the sum of parallel and perpendicular forces:

$$
\mathbf{F}^{*}=\left(\mathbf{F}_{\|}\right)^{*}+\left(\mathbf{F}_{\perp}\right)^{*}
$$

Usually, the term with the $\gamma$ derivative is eliminated by means of not fully justified arguments, so arriving to the following equation from reference [3], an equation conserved in even more recent books:

$$
\mathbf{F}^{*}=\mathbf{F}_{\perp}=\frac{\mathrm{M} \gamma \mathrm{d} \mathbf{v}}{\mathrm{dt}}=\mathrm{M} \gamma \mathbf{a}_{\perp}
$$

This model has important drawbacks: one is to have cancelled one term that had been properly obtained. It could be justified by assuming that the $\gamma$ factor is a constant though, in general, it is given as a time function by Equation (7a). To define the necessary precision of $\gamma$, the expansion in power series helps, by means of the first 3 terms applied to Equation (7a):

$$
\gamma=1+(1 / 2) \beta^{2}+(1 / 2)(3 / 4) \beta^{4}
$$

A substitution of the 2 first terms of (11) in (7a), gives a time derivative:

$$
\frac{\mathrm{d} \gamma}{\mathrm{dt}}=\frac{1}{2 \mathrm{c}^{2}}\left(\frac{\mathrm{dv^{2 }}}{\mathrm{dt}}\right)=\frac{1}{2 \mathrm{c}^{2}} 2 \mathrm{v} \frac{\mathrm{dv}}{\mathrm{dt}}=\mathrm{va} / \mathrm{c}^{2}
$$

So,

$$
\operatorname{Mv} \frac{\mathrm{d} \gamma}{\mathrm{dt}}=\mathrm{Mv} \frac{\mathbf{v a}}{\mathbf{c}^{2}}=\mathrm{Mv}^{2} \frac{\mathbf{a}}{\mathbf{c}^{2}}=\operatorname{Ma} \beta^{2}
$$

Substitution of Equations (10) and (13) in Equation (9) gives:

$$
\mathrm{F}^{*}=\operatorname{Ma} \gamma+\operatorname{Ma} \beta^{2}=\operatorname{Ma}\left(\gamma+\beta^{2}\right)
$$

In the Hubble field, in a co-moving coordinate, it would be assumed as:

$$
\mathbf{F}_{\mathbf{H}}=\mathrm{M} \Gamma_{\mathrm{H}}=\mathrm{MH}^{2} \mathbf{r}
$$

Equation (14) shows that, at the highest velocities $(\beta>0.9)$ the $\beta^{2}$ value represents more than $25 \%$ of the relativistic factor (the parenthesis in Equation (14)). At low velocities $(\beta<0.1)$, the $\beta^{2}$ percentage falls to less than $1 \%$. Otherwise, if it would be applied the third term of the $\gamma$ expansion, the percentage from the $\beta^{4}$ term in the relativistic factor would be even lower than $0.01 \%$.

Another drawback in the model of Equation (10) is that, in the interaction of two relativistic forces $\mathbf{F}_{\mathrm{a}}{ }^{*}$ and $\mathbf{F}_{\mathrm{b}}{ }^{*}$ it is assumed that, in practice, they only would act the transverse components (since the parallel ones have been ruled out) independently of their magnitudes and their angles with respect to the $\mathrm{X}$ axis. Such a case would not be accurate enough to determine the corresponding work developed by one force and the potential energy accumulated in the other mass. 
The supporting example of Equation (10), mentioned in reference [3] as an Einstein equation, should be rather related to a different case: the Lorentz force in e. m. with a final circular trajectory.

Anyway, in the classical and the Special Relativity models, the work would correspond to an increase in the potential energy of the mass M. In the Hubble model it may be different because there is not any known physical agent that generates the Hubble force. This one manifests only if a mass is present (similar, in this respect, to the gravitational case). Therefore there are 3 equations to express the Hubble force: the above named (1) and (2a) to determine the Hubble acceleration and, by similitude to Equation (5), the one here named as Equation (15). Otherwise, the force produced by the Universal gravitational acceleration $\Gamma_{\mathrm{G}}$ if it acts on an external mass $\mathrm{M}$, would be:

$$
\mathbf{F}_{\mathrm{G}}=\mathrm{M} \Gamma_{\mathrm{G}}
$$

Really, masses move away with an acceleration $\Gamma_{\mathrm{H}}$ since they are precisely placed in their co-moving coordinates in the Universe, while this one expands (it means that $\mathbf{r}$ increases but $\theta$ and $\phi$ remain constant); so, as $\mathbf{r}$ grows, the Hubble parameter decreases accordingly to Equation (2a). It must be emphasized that $\boldsymbol{\Gamma}_{\mathrm{H}}$ is a constant but $\boldsymbol{\Gamma}_{\mathrm{G}}$ is inversely proportional to $\boldsymbol{r}^{2}$ since it is given by:

$$
\Gamma_{G}=\mathrm{GM} / \mathbf{r}^{2}
$$

\section{The Energy in the Hubble Field}

It has been assumed in previous paragraphs that the Hubble force should not necessarily be expressed by means of mass since the Hubble potential exists in the observable Universe and, probably, it does too in the external empty space. Then, as previously noted, the force may be better expressed by means of the intensity of the Hubble field $\boldsymbol{\Gamma}_{\mathrm{H}}$, i.e., the gradient of the Hubble potential of Equation (1).

In a previous example, in the Appendix of reference [2] it was shown that, if the value of the constant Universe acceleration $\boldsymbol{\Gamma}_{\mathrm{H}}$ would be experimentally confirmed, the Hubble potential could be calculated as:

$$
\mathrm{V}_{\mathrm{H}}=\int_{\mathrm{o}}^{\mathrm{r}} \Gamma_{\mathrm{H}} \mathrm{dr}=\int_{\mathrm{o}}^{\mathrm{r}} \mathrm{H}^{2} \mathrm{r} \cdot \mathrm{dr}=\mathrm{H}^{2} \mathrm{r}^{2} / 2
$$

In the Hubble field, kinetic and potential energies always have identical and positive magnitudes so showing that it is not adequate to assume a total similitude with the gravitational field. The Hubble potential exists at every point $\mathbf{r}$ in the Universe and, if a mass would be placed in there, its potential energy would instantaneously manifest as kinetic energy, all of them expressed as:

$$
\mathrm{U}_{\mathrm{H}}=\mathrm{MV}_{\mathrm{H}}=\frac{\mathrm{MH}^{2} \mathrm{r}^{2}}{2}=\mathrm{K}_{\mathrm{H}}
$$

\section{A De Sitter-Hubble Equation}

In reference [4] it had been reminded an equation that included the constant $\Lambda$, 
added by Einstein in his gravitational equation, not as a universal constant but as a necessary constant of integration [5]:

$$
\mathrm{R}_{\mu v}-\frac{\mathrm{g}_{\mu v} \mathrm{R}}{2}-\mathrm{g}_{\mu v} \Lambda=8 \pi \mathrm{GT}_{\mu \nu}
$$

$G$ is the universal gravitational constant, $T_{\mu \nu}$ is the energy-momentum tensor and $g_{\mu \nu}$ is the metric tensor. The first two terms on the left side are known as the Einstein tensor $\mathrm{G}_{\mu v}$.

De Sitter assumed a Universe with no matter or radiation [4]; it means that $\mathrm{T}_{\mu \nu}=0$. Given the very low density in the Universe and the assumed 0 density in the exterior, Equation (19) may be written as:

$$
\mathrm{R}_{\mu \nu}-\frac{\mathrm{g}_{\mu \nu} \mathrm{R}}{2}=\mathrm{g}_{\mu v} \Lambda
$$

So, a definition of a $\Lambda$ tensor would be:

$$
\left(\mathrm{T}_{\mu \nu}\right)_{\Lambda}=\mathrm{g}_{\mu \nu} \Lambda
$$

The Einstein tensor must always remain, since it expresses the space-time curvature originated by the mass of the Universe.

Equation (2.9) of reference [1] gave the relation between $\Lambda$ and $H$, as:

$$
\Lambda=3 H^{2}
$$

which could have also been derived from reference [6], Equation (143.4), as a function of the Universe radius $\mathrm{R}$, if it is assumed $c=1$ :

$$
\frac{1}{\mathrm{R}^{2}}=\frac{\Lambda}{3}
$$

Similarly to Equation (21), a Hubble tensor would be:

$$
\left(\mathrm{T}_{\mu v}\right)_{\mathrm{H}}=\mathrm{g}_{\mu \nu} 3 \mathrm{H}^{2}
$$

So, the scalar of the Hubble tensor is:

$$
\mathrm{T}_{\mathrm{H}}=3 \mathrm{H}^{2}=\Lambda\left(\mathrm{s}^{-2}\right)
$$

which results equal to the Ricci scalar given by reference [5]. Therefore, it is possible to write:

$$
\mathrm{G}_{\mu \nu}=\mathrm{R}_{\mu \nu}-\frac{\mathrm{g}_{\mu \nu} \mathrm{R}}{2}=\mathrm{g}_{\mu \nu} 3 \mathrm{H}^{2}
$$

A simple form to express a De Sitter-Hubble equation would be:

$$
\mathrm{G}_{\mu v}=\left(\mathrm{T}_{\mu v}\right)_{\mathrm{H}}
$$

The properties of the fundamental metric tensor $\mathrm{g}_{\mu \nu}$ of the Hubble tensor have been defined in reference [4], and others, by means of its matrix expression obtained from the coefficients of the RWFL equation.

\section{Some Comments on the Search for Symmetry in Science}

The general criterion about symmetry is that of a mirror image though, in the 
physical science, symmetry means different: the constancy of a feature faced to a change of coordinates. In this paragraph both criteria are considered. Symmetry is an important concept postulated by references as relevant as E.A. Poe [7] ("Symmetry and consistency are convertible terms") and L. Lederman [8] ("Symmetry...was the last lesson of the twentieth century"). As an example, the Hubble force, as opposed to the gravitational force, would represent an eventual example of mirror Symmetry in the Physical Universe.

Many important papers have been written about Symmetry in Science, as the one including the Mathematics of E. Noether, H. Weyl and P. Wigner, in a book by K. Bradding [9] and, in the Chemical area, the work of P. Curie [10]. As well, reference [5] applied a Symmetry condition to tensors in Relativity. The Super Symmetry project (SUSY) started at the end of $20^{\text {th }}$ century though it was mainly oriented to the nuclear level. However, there is not a case where it would have been found a symmetric mirror (at a macroscopic level) for a fundamental physical entity: the time. R. Feynmann performed an analysis of (Parity, Charge and Time) symmetries of physical law [11], a necessary concept for the nuclear structure (into the limits of the Heisenberg principle). R. Penrose [12] refers to time inversion in nuclear reactions and reference [13] mentions the possibility of time reversal in a travel through the space-time diagrams (if the traveler would reach a speed higher than c)that anyway would imply a violation to the causality principle. Otherwise, from Aristotle to Saint Augustin and Newton and from Eddington to Hawking, they have developed very deep concepts on time. As well, accordingly to the $3^{\text {rd }}$ Law of Thermodynamics, a decrease in entropy could imply a time inversion though, at present, it does not look as a feasible reality.

Besides, it is in the theory of Relativity that Symmetry would also appear by its postulate of law's invariance so allowing, in some equations, the interchange of signs \pm in the time parameter. In addition it must be said that, by the above assumed allocation of matter in co-moving coordinates in the Universe expansion, it cannot be there applied the concepts of inertial and non-inertial frames of reference, so permitting a feasible value of $\mathbf{v}_{\mathrm{H}}>\mathbf{c}$, as it has been assumed in reference [13]. A very important concept of time in Physics is the proper time $\tau$ defined by the inertial frame equation:

$$
\tau=\mathrm{t}_{\mathrm{c}} / \gamma
$$

where $t_{c}$ is the coordinate time and $\gamma$ was defined in Equation (7a); so, if $\mathbf{v} \rightarrow \mathbf{c}, \tau$ $\rightarrow 0$. Even in the case that $\mathbf{v}>\mathbf{c}$, $\tau$ will not appear as a negative time but an imaginary time, as it occurs at the present radial Universe function $r_{o}$. Furthermore, the proper time obtained by the non-inertial frame equation

$$
\tau=\mathrm{t}_{\mathrm{o}}\left(1-\mathrm{GM}_{\mathrm{U}} / \mathrm{cr}_{\mathrm{o}}^{2}\right)^{-1 / 2}
$$

gives an imaginary value but not a negative one. If it would be necessary to work with such an imaginary time, it could be intended by means of the inverse Laplace transform. Therefore, by the above mentioned examples, time is assumed symmetric in Physics, though not in the mirror image sense. Besides, the Hubble 
parameter $H$, experimentally measured as a positive one [14], keeps its sign independently of the sign of time.

\section{Conclusions}

\subsection{From Previous Articles [1] and [2]}

1) The intensity of the Hubble field is defined as the positive gradient of a positive potential, Equation (1). This intensity represents the Hubble acceleration for the Universe expansion, a constant $\boldsymbol{\Gamma}_{\mathrm{H}}=\mathrm{H}^{2} \mathbf{r}\left(\mathrm{m} \cdot \mathrm{s}^{-2}\right)$.

2) It had been deduced [2] that the Lagrangian

$$
\mathrm{L}_{\mathrm{H}}=\mathrm{K}_{\mathrm{H}}-\mathrm{U}_{\mathrm{H}}=0
$$

due to the sameness of both energy magnitudes. As well, the generalized time derivative of $\mathcal{H}$ in ref. ([2], Equation (2.11)) would be 0; i.e.,

$$
\mathcal{H}=\text { constant }
$$

3) It had been shown in paragraph 3.2 of reference [2] that, at any $r$ position in the Universe, the positive Hubble potential energy is equal to the Hubble kinetic energy so showing a total difference with the gravitational energy.

4) It has been proposed a Hubble tensor, in a similar form to that of ref. [4] for $\Lambda$, as:

$$
\left(\mathrm{T}_{\mu v}\right)_{\mathrm{H}}=\mathrm{g}_{\mu \nu} 3 \mathrm{H}^{2}
$$

\subsection{From the Present Article}

1) It has been assumed that the Hubble field is a scalar, stationary and positive field since, at every position $\mathbf{r}$, it is possible to determine a positive scalar potential $\mathrm{V}_{\mathrm{H}}$, Equation (1).

2) It has proposed a correction for the equation of the Force in Special Relativity (Equation (14)).

3) It proposed the following equation for the energies of a mass $M$ if it is placed at a co-moving coordinate $(\mathbf{r}, \Theta, \phi)$ in the Hubble field:

$$
\mathrm{U}_{\mathrm{H}}=\mathrm{M} \cdot \Gamma_{\mathrm{U}} \cdot \mathbf{r} / 2=\mathrm{K}_{\mathrm{H}}
$$

4) It is possible to assume a De Sitter-Hubble equation, for an expanding Universe, as: $G_{\mu \nu}=\left(T_{\mu \nu}\right)_{H}(26)$. The physical meaning of the Hubble scalar shows that the surface expansion acceleration is $⿻ 5 \times 10^{17}\left(\mathrm{~m}^{2} / \mathrm{s}^{2}\right)$.

5) Respect to time Symmetry, some conclusions could be:

- It is evident that, at the critical time, the Hubble acceleration $\Gamma_{H}$ would be an exact, though brief, mirror of the gravitational intensity $\Gamma_{G}$; i.e., its symmetrical image (Figure 1 ).

- Besides, as far as the Hubble field would remain, the Big Crunch will never occur, even in the case of time reverts since, as far as $\mathbf{r}$ keeps growing, $\boldsymbol{\Gamma}_{\mathrm{H}}>\boldsymbol{\Gamma}_{\mathrm{G}}$ after the critical rime $\mathrm{t}_{\mathrm{c}}$.

- As an apology, it could be suitable to recall a phrase of reference [15]: "For the treatment of the whole Universe... We can only relay on the 
roughest methods of scientific induction".

\section{Acknowledgements}

The author is grateful to M.Sc. A. Zúñiga for his kind assistance in the realization of this work.

\section{References}

[1] Lartigue, J. (2016) Journal of Modern Physics, 7, 1607-1615. https://doi.org/10.4236/jmp.2016.712145

[2] Lartigue, J. (2017) Journal of Modern Physics, 8, 2159-2166. https://doi.org/10.4236/jmp.2017.813131

[3] Katz, R. (1964) An Introduction to the Special Theory of Relativity. D. van Nostrand Co., Los Angeles, 55.

[4] Bergstrom, L. and Goobar, A. (2004) Cosmology and Particle Astrophysics. 2nd Edition, Springer-Berlin, 59-60.

[5] Einstein, A., et al. (1923) The Principle of Relativity. Dover, New York, 195-196.

[6] Tolman, R. (1958) Relativity and Thermodynamics. Clarendon Press, Oxford, 349.

[7] Poe, E.A. (1997) Eureka. Prometeus Books, New York, 80-81.

[8] Lederman, L. and Hill, T. (2007) Symmetry and the Beautiful Universe. Prometeus Books, New York, 20.

[9] Brading, K. and Castellani, E. (2003) Symmetries in Physics. Cambridge University Press, Cambridge, 5-6. https://doi.org/10.1017/CBO9780511535369

[10] Shubnikov, A. (1988) Computers \& Mathematics with Applications, 16, 357.

[11] Feynmann, R. (1994) The character Physical Law. Modern Library, New York, Ch. 4, p. 84 .

[12] Penrose, R. (1979) Singularities and Time Assimetry. Cambridge, p. 631.

[13] Sartory, L. (1996) Understanding Relativity. UCLA, Berkeley, p. 147.

[14] Hinshaw, G. (2013) NASA/WMAP, Science Team Report, 03/05/2013.

[15] Tolman, R. op cit, p. 487. 